
Kidney
Blood Pressure
Research

Kidney Blood Press Res 2018;43:1772-1785

DOI: $10.1159 / 000495635$

Published online: 1 December 2018

(c) 2018 The Author(s)

Published by S. Karger AG, Basel

Accepted: 21 November 2018

\title{
The Kidney Specific Protein myo-Inositol Oxygenase, a Potential Biomarker for Diabetic Nephropathy
}

\author{
Peng Gao ${ }^{\mathrm{a}}$ Bo Xu $\mathrm{Xu}^{\mathrm{a}}$ Panai Song ${ }^{\mathrm{a}}$ Xuejing Zhu ${ }^{\mathrm{a}}$ Shuguang Yuan ${ }^{\mathrm{a}}$ \\ Yashipal S Kanwarb Lin Sun ${ }^{a}$ \\ aDepartment of Nephrology, The Second Xiangya Hospital, Central South University, Hunan Key \\ Laboratory of Kidney Disease and Blood Purification, Hunan, China, 'D Departments of Pathology \\ \&Medicine, Northwestern University, Chicago, USA
}

\section{Key Words}

myo-inositol oxygenase $\cdot$ Diabetic nephropathy $\cdot$ Tubular damage $・$ Biomarker

\begin{abstract}
Background/Aims: Renal tubular injury plays an important role in the progression of diabetic nephropathy (DN). However, there is a lack of specific biomarkers for tubular damage in incipient DN. We have evaluated the role of myo-inositol oxygenase (MIOX) in the tubular injury of DN, but whether it could serve as a new biomarker for the early diagnosis of DN is unclear. Methods: Ninety patients with type 2 diabetes mellitus (T2DM) were divided into normoalbuminuria, microalbuminuria and macroalbuminuria groups. Fifteen patients from the last group were pathologically diagnosed as type 2 DN (T2DN), and fifteen patients with minimal change disease served as a control group. The expression of MIOX and silent information regulator 1 (Sirt1) in renal biopsies was determined by immunohistochemistry $(\mathrm{IHC})$, and serum/urine MIOX, Sirt1, KIM-1 and NGAL were measured using enzyme-linked immunosorbent assays (ELISAs). Spearman's correlation and multiple regression analyses were carried out for statistical analyses. Results: Compared with the controls, MIOX expression was significantly increased in the renal tissues of T2DN patients, and was positively correlated with tubulointerstitial lesions and renal ROS production but inversely correlated with Sirt1 expression. In addition, the serum and urine MIOX were significantly increased and gradually elevated with the increasing of UACR. Interestingly, elevated MIOX levels in serum and urine were found in diabetic patients without early signs of glomerular damage (normoalbuminuric group). Further multivariate regression analysis showed that sMIOX and UMIOX correlated significantly with $\mathrm{HbA} 1 \mathrm{c}$, serum creatinine and logUACR, respectively. Conclusion: These data indicate that increased MIOX expression in the kidney contributes to tubular damage in DN. The concentration of MIOX in the serum and urine may serve as a new biomarker for the early diagnosis of DN.




\section{Kidney Blood Pressure Research}

\section{Introduction}

Diabetic nephropathy (DN) is a serious microvascular disease in patients with diabetes mellitus (DM) that ultimately leads to progressive renal failure [1]. The natural evolution of DN has been classically divided into three stages: normoalbuminuria, microalbuminuria, and macroalbuminuria [2]. In addition, early-stage DN is characterized by normoalbuminuria, also called incipient DN [3]. Diagnosis of DN at the incipient stage would be critical and thus allow time for intensive therapeutic interventions that could reduce morbidity and mortality. Traditional studies to unravel the pathogenesis and pathophysiology of DN have mostly focused on the glomerulus [4], which clinically manifests as hyperfiltration and microalbuminuria [1]. However, recent investigations have found that the renal tubulointerstitial compartment also seems to play an equally important role in the pathogenesis of DN, since it is persistently exposed to various metabolic- and hemodynamicinjuring factors, such as high glucose [5]. Thus, tubular cells are not only affected secondary to glomerular injury but are also primary targets for pathological influences in diabetes [6]. It has been shown that renal tubular damage can precede albumin excretion in the absence of glomerular lesion [7]. Well-described characteristic tubulointerstitial histologic changes include tubular hypertrophy followed by thickening of the tubular basement membrane and interstitial fibrosis [8]. As a result, tubulointerstitial injury is also a major feature of DN and could be an early manifestation of renal dysfunction [9].

After tubular injury occurs, proximal tubular proteins may leak into the tubular space and be excreted into urine or reabsorbed into blood. Therefore, many studies have been looking for chemical substances that have been released into serum or urine in order to find a biomarker for the early diagnosis of tubular injury at the incipient stage. Many biomarkers, such as neutrophil gelatinase associated lipocalin (NGAL), kidney injury molecule 1 (KIM1), and liver-fatty acid-binding protein (LFABP) have been proposed for the diagnosis or monitoring of diabetic complications, particularly kidney disease [10]. However, the sensitivity of these markers in detecting tubular injury of DN patients requires further investigation.

MIOX, a 33-kDa nonheme iron protein, is considered to be the key regulator of myoinositol (MI) levels $[11,12]$, which catabolizes MI into D-glucuronic acid exclusively in the kidney [13]. Previously, our laboratory cloned a renal-specific oxido-reductase gene from a human cDNA library and initially designated it as RSOR [14]. Now, it has been uniformly named as myo-inositol oxygenase (MIOX) [11]. We determined that MIOX expression is confined to renal proximal tubular cells and found that it is upregulated in the kidney of diabetic mouse. Current researches have shown that MIOX not only regulates MI metabolism but also participates in the pathogenesis of DN tubular injury through non-MI metabolic pathways, such as by stimulating the release of TGF $\beta$ [15], disrupting the redox status [16] and regulating mitochondrial dynamics [17]. Interestingly, Gaut et al. found that an increase in serum MIOX preceded the elevation of serum creatinine in patients with acute kidney injury (AKI), which was closely associated with the degree of tubular damage [18], indicating that serum MIOX can be used as an AKI biomarker. Despite the evident importance of MIOX in streptozotocin-induced diabetic mice tubular injury, little is known about its role and diagnostic value in DN patients.

In light of the above considerations, we first investigated MIOX expression in the kidney tissues of T2DN patients, then analyzed serum and urine MIOX levels in T2DM patients with different stages of UACR in order to assess the potential relationships between this tubular biomarker and the severity of renal involvement. 


\section{Kidney Blood Pressure Research}

\section{Materials and Methods}

\section{Participants}

Ninety patients were recruited from the Second Xiangya Hospital, Central South University, who were newly diagnosed with T2DM, according to the American Diabetes Association 2010 diagnostic criteria. Patients with the following were excluded: those using adrenal cortical hormones, immunosuppression drugs or RAS inhibitors and those with urinary tract infections, or with inflammatory, neoplastic, cardiovascular, hepatic, renal, lung or neuroendocrine diseases. Patients with T2DM were classified into three groups, according to their urinary albumin/creatinine ratio (UACR): the normoalbuminuric group (UACR $<30 \mathrm{mg} / \mathrm{g}, n=30$ ); the microalbuminuric group (UACR $30-300 \mathrm{mg} / \mathrm{g}, n=30$ ) and the macroalbuminuric group (UACR $>300 \mathrm{mg} / \mathrm{g}$, $n=30$ ). All of the patients in the normoalbuminuric and microalbuminuric groups were newly diagnosed with T2DM and T2DN, respectively. And there were fifteen patients in the macroalbuminuria group that were diagnosed with T2DN by renal biopsy. In order to access the histological changes and the expression of MIOX and Sirt1 in the renal biopsies, fifteen patients with minimal change disease (MCD) who were wellmatched with patients with T2DN with respect to age, gender, BMI, and course of disease were enrolled as a pathologic control. Thirty age and sex-matched healthy subjects were enrolled as a control group after a careful history and clinical examination. The institutional review board and the administrators of the Department of Nephrology in the Second Xiangya Hospital approved the protocol for this study. Informed consent was obtained from all of the participants.

\section{Collection of serum and urine sample}

Fasting blood samples were tested for creatinine, fasting blood glucose (FBG), glycosylated hemoglobin (HbA1c), and other chemical laboratory parameters. First-voided morning urine samples were collected and centrifuged at $2500 \mathrm{rpm}$ for $10 \mathrm{~min}$, then urine aliquots were stored at $-80^{\circ} \mathrm{C}$ until analysis.

\section{Biochemical analysis of sample}

The liver and renal functional parameters, including the triglyceride (TG), total cholesterol (TC), FBG, uric acid (UA), and serum creatinine (Scr) levels were measured by automatic picric colorimetry (Hitachi 912 automated analyzer, Roche, USA). In addition, HbA1c level were determined by automatic highperformance liquid chromatography (HPLC) (VARIANT-11 Hemoglobin Testing System; Bio-Rad, Hercules, CA). eGFR (mL/min/1.73 $\mathrm{m}^{2}$ ) was estimated using the Modification of Diet in Renal disease (MDRD) formula (for Chinese): $175 \times \operatorname{Scr}(\mathrm{mg} / \mathrm{dL})^{-1.234} \times$ Age (in years) ${ }^{-0.179}$ (if female $\times 0.79$ ). Urinary microalbumin was measured by immune turbidimetry (Beckman Coulter, USA) and UACR was calculated.

\section{Morphological analysis of kidney}

Formalin-fixed paraffin embedded human kidney tissue were obtained from the Department of Pathology at Second Xiangya Hospital. The 4- $\mu$ m paraffin sections from kidney biopsies were stained with hematoxylin-eosin staining (HE), periodic acid Schiff (PAS), periodic acid-silver methenamine (PASM) and Masson. The tubulointerstitial lesion index was determined by using a semiquantitative scoring system [19]. Tubular injury was scored as previously described [20].

\section{Immunohistochemistry (IHC)}

Renal biopsy tissue was paraffin-embedded and sectioned. After de-paraffinization, the sections were incubated with $3 \% \mathrm{H}_{2} \mathrm{O}_{2}$ solution to block endogenous peroxidase. Antigen retrieval was carried out using $0.1 \mathrm{M}$ sodium citrate (pH 6.0) for $60 \mathrm{~min}$. Sections were incubated with anti-MIOX antibody (1:100, abcam) or anti-Sirt1 antibody (1:100, CUSABIO) overnight at $4^{\circ} \mathrm{C}$, and horseradish peroxidase-conjugated secondary antibody and diaminobenzidine (DAB) substrate were added sequentially. After hematoxylin counter staining and dehydration, the sections were mounted and observed under a Leica microscope.

\section{Detection of ROS}

Intracellular ROS production in renal tissues was measured using $4 \mu \mathrm{m}$ cryostat sections that were stained with Dihydroethidium (DHE) (1:20000, Sigma-Aldrich) and incubated at $37^{\circ} \mathrm{C}$ for $20 \mathrm{~min}$. Twenty 


\section{Kidney Blood Pressure Research}

randomly selected fields were photographed. The color threshold was kept constant for each image and the mean fluorescence intensity was calculated.

\section{Determination of KIM-1, NAGL, MIOX and Sirt1 protein levels}

Serum/urine samples from the subjects were analyzed for KIM-1, NAGL, MIOX and Sirt1 protein levels using enzyme-linked immunosorbent assay (ELISA) kits from USCN Life Science (Catalog no: SEA785Hu for KIM-1, SEB388Hu for NGAL, SEG831Hu for MIOX and E94912Hu for SIRT1, Wuhan/CHINA). Briefly, standards and samples $(100 \mu \mathrm{L} /$ well $)$ were pipetted into a 96-wells plate where anti-KIM-1/anti-NGAL/ anti-MIOX/anti-Sirt1 has been pre-coated at ambient temperature for 1 hour. After incubation, the substrate solution was added and the plates were incubated for 10-20 min. Then, adding the stop solution to terminated the reaction. Finally, the intensity of the color change in each well was measured at $450 \mathrm{~nm}$ in a microplate reader (Chromate Manager 4300, Palm City, USA).

\section{Statistical analysis}

All statistical analyses were performed using the SPSS (22.0) software, MedCalc (8.0), and GraphPad Prism software (7.0). Data are expressed as mean \pm SD when normally distributed, and as median and QR (quartile range) in case of skewed distribution. Differences between two groups were tested using t-test or Mann-Whitney U test. A One-way ANOVA or Kruskale-Wallis $\mathrm{H}$ test was used for multiple independent sample. Spearman's correlation and multivariate regression analysis were calculated between the renal MIOX expression, sMIOX, uMIOX, and other clinical parameters. Receiver operating characteristics curve (ROC) analysis was performed to calculate the area under the curve (AUC) for sMIOX and uMIOX to find an optimal cutoff value capable of identifying diabetic patients with normal albumin excretion. $p<0.05$ was defined to be statistically significant.

\section{Results}

Overall characteristics of study subjects

The clinical characteristics of the healthy subjects and patients with T2DM are summarized in Table 1. There was no difference in age, gender, BMI or uric acid levels among the four groups. Diabetes duration, FBG, HbA1c and triglyceride levels of the T2DM patients were significantly higher than those in the control group $(\mathrm{p}<0.05)$. In addition, FBG, HbA1c, SBP, and UACR in the microalbuminuric and macroalbuminuric group were increased, compared with those in normoalbuminuric group $(\mathrm{p}<0.05)$. Additionally, the values of FBG, HbA1c, triglyceride, total cholesterol, Scr and UACR in the macroalbuminuric group were significantly higher than those in microalbuminuria group $(p<0.05)$. In contrast, the serum albumin and eGFR in macroalbuminuria group were dramatically decreased compared with other groups $(\mathrm{p}<0.05)$.

Pathological lesions and tubular interstitial damage score are correlated with renal MIOX expression and oxidative stress

The detailed clinical features of patients with MCD are shown in Table 2. Morphological lesions in renal tissues were accessed by HE, PASM, PAS and Masson staining. Light microscopic analysis showed that samples from T2DN had glomerular basement membrane (GBM) thickening, mild mesangial expansion and extracellular matrix accumulation (Fig. 1A b, d, f, h), while the control group showed no obvious glomerular lesions (Fig. 1A a, c, e, g). Then, tubulointerstitial lesion index and tubular damage scoring system were determined. The control group mostly had scores of $0-1$ points, while scores of 2 to 5 points were observed for patients with T2DN (Fig. 1B). Further analysis showed that tubular interstitial damage was positively correlated with renal MIOX expression (Fig. $2 \mathrm{~A}, \mathrm{r}=0.665$ ) and the renal ROS production (Fig. 2B, $r=0.588$ ), while inversely related to Sirt1expression (Fig. 2C, $r=-0.578$ ). Furthermore, multiple regression analysis found a significant correlation among tubular interstitial damage, renal MIOX expression, oxidative stress and Sirt1 expression. The 


\section{Kidney Blood Pressure Research}

Table 1. Clinical characteristics of participants Abbreviations: N/A, not applicable; HS, health subject; $y$, years; m, male; f, female; kg, kilogram; FBG, fasting blood glucose; HbA1c, glycosylated hemoglobin; SBP, systolic blood pressure; DBP, diastolic blood pressure; Alb, albumin; eGFR: estimated glomerular filtration rate; UACR, urine albumin/creatinine ratio; uKIM-1: urinary kidney injury molecule 1; uNGAL: urinary neutrophil gelatinase-associated lipocalin; sMIOX, serum MIOX; uMIOX, urinary MIOX; Data are expressed as means \pm SD except for UACR, KIM-1, NGAL: median (25-75th percentile). ${ }^{1} \mathrm{p}<0.05$ versus. Health subjects; ${ }^{2} \mathrm{p}<0.05$ versus. normoalbuminuria; ${ }^{3} \mathrm{p}<0.05$ versus. microalbuminuria

\begin{tabular}{|c|c|c|c|c|}
\hline Parameter & $\begin{array}{l}\text { Health subjects }(\mathrm{n}=30) \\
(\mathrm{n}=30)\end{array}$ & Normoalbuminuria $(\mathrm{n}=30)$ & $\begin{array}{c}\text { Patients with T2DM } \\
\text { Microalbuminuria }(\mathrm{n}=30)\end{array}$ & Macroalbuminuria $(n=30)$ \\
\hline Age (y) & $48.1 \pm 6.8$ & $50.1 \pm 7.2$ & $49.3 \pm 6.6$ & $51.3 \pm 5.8$ \\
\hline Diabetes duration (y) & N/A & $8.4 \pm 2.7$ & $8.8 \pm 3.0$ & $8.8 \pm 2.8$ \\
\hline $\operatorname{Sex}(m / f)$ & $15 / 15$ & $16 / 14$ & $16 / 14$ & $17 / 13$ \\
\hline $\operatorname{BMI}\left(\mathrm{kg} / \mathrm{m}^{2}\right)$ & $23.3 \pm 1.9$ & $23.1 \pm 2.5$ & $24.5 \pm 2.6$ & $24.4 \pm 2.8$ \\
\hline FBG $(\mathrm{mmol} / \mathrm{l})$ & $4.8 \pm 0.9$ & $7.0 \pm 2.0^{1}$ & $7.8 \pm 2.3^{1,2}$ & $8.6 \pm 2.2^{1,2,3}$ \\
\hline $\mathrm{HbA} 1 \mathrm{c} \%(\mathrm{mmol} / \mathrm{mol})$ & $\begin{array}{c}3.9 \pm 0.7 \\
(18.8 \pm 8.1)\end{array}$ & $\begin{array}{c}7.7 \pm 1.5^{1} \\
(60.5 \pm 16.4)^{1}\end{array}$ & $\begin{array}{c}8.6 \pm 1.6^{1,2} \\
(66.2 \pm 16.2)^{1,2}\end{array}$ & $\begin{array}{c}9.6 \pm 2.3^{1,2,3} \\
(81.6+25.2)^{1,2,3}\end{array}$ \\
\hline SBP (mmHg) & $110.1 \pm 6.9$ & $122.5 \pm 9.0^{1}$ & $135.8 \pm 10.8^{1,2}$ & $136.7 \pm 10.0^{1,2}$ \\
\hline DBP (mmHg) & $69.9 \pm 6.7$ & $77.1 \pm 7.3$ & $78.7 \pm 6.5$ & $80.1 \pm 8.3^{1}$ \\
\hline Serum albumin $(\mathrm{g} / \mathrm{L})$ & $39.0 \pm 2.0$ & $37.5 \pm 3.6$ & $38.0 \pm 4.2$ & $31.8 \pm 7.11,2,3$ \\
\hline Triglyceride (mmol/L) & $1.1 \pm 0.3$ & $1.5 \pm 0.6^{1}$ & $1.5 \pm 0.71$ & $2.4 \pm 1.6^{1,2,3}$ \\
\hline Total cholesterol (mmol/l) & $4.4 \pm 0.7$ & $4.3 \pm 1.0$ & $4.2 \pm 1.2$ & $5.2 \pm 1.6^{1,2,3}$ \\
\hline eGFR $\left(\mathrm{ml} / \mathrm{min} / 1.73 \mathrm{~m}^{2}\right)$ & $112.9 \pm 11.6$ & $109.4 \pm 11.6$ & $108.3 \pm 10.3$ & $85.3 \pm 10.4^{1,2,3}$ \\
\hline Serum creatinine $(\mu \mathrm{mol} / \mathrm{l})$ & $54.7 \pm 11.8$ & $57.3 \pm 9.5$ & $59.4 \pm 11.1$ & $137.0 \pm 41.8^{1,2,3}$ \\
\hline Uric acid $(\mu \mathrm{mol} / \mathrm{L})$ & $281.2 \pm 59.0$ & $281.2 \pm 69.5$ & $281.8 \pm 74.7$ & $285.3 \pm 63.5$ \\
\hline UACR (mg/g) & $5.2(3.7-6.3)$ & $15.6(10.3-25.5)^{1}$ & $52.5 .6(31.8-79.6)^{1,2}$ & $1932.3(578.6-3318.6)^{1,2,3}$ \\
\hline Sirt1 (ng/ml) & $9.7 \pm 2.8$ & $8.4 \pm 3.3^{1}$ & $6.6 \pm 2.5^{1,2}$ & $4.8 \pm 2.91,2,3$ \\
\hline uKIM-1/urine Cr (ng/ mg) & $0.7(0.6-1.0)$ & $0.8(0.5-1.3)$ & $1.7(0.8-2.3)^{1,2}$ & $4.1(1.9-6.3)^{1,2,3}$ \\
\hline uNGAL/urine Cr (pg/mg) & $7.2(6.2-17.3)$ & $6.8(5.7-20.5)$ & $7.9(6.3-37.8)^{1,2}$ & $16.9(9.3-73.5)^{1,2,3}$ \\
\hline sMIOX (pg/ml) & $220.0 \pm 66.7$ & $530.9 \pm 131.2^{1}$ & $729.6 \pm 160.4^{1,2}$ & $1557.4 \pm 420.1^{1,2,3}$ \\
\hline uMIOX/urine $\mathrm{Cr}$ (pg/mg) & $252.9 \pm 44.6$ & $679.3 \pm 217.7^{1}$ & $921.9 \pm 155.0^{1,2}$ & $1621.4 \pm 433.4^{1,2,3}$ \\
\hline
\end{tabular}

regression coefficients of MIOX ( $\beta 1)$, ROS ( $\beta 2)$ and Sirt1 ( $\beta 3)$ contributed to tubular interstitial injury was 0.425 , -0.325 and 0.089 , respectively $(\mathrm{p}<0.05$ for all).

Renal expression of MIOX is increased in patients with T2DN and is correlated with oxidative stress and Sirt1 expression

IHC staining revealed elevated MIOX expression in T2DN patients, compared with the control (Fig. 1C $b$ vs a). Moreover, the increase in staining was predominantly confined to the renal proximal tubules, and very little expression was observed in the glomerular mesangium. In contrast, compare with the control group, Sirt1 expression was decreased in patients with T2DN (Fig. 1C d vs c). In addition, DHE staining, which reflects the intracellular ROS production, was performed to access oxidative stress. Compared to the control group, significantly higher ROS levels were observed in the renal tissues of patients with T2DN (Fig. 1C f vs e). Quantitative analysis showed that MIOX expression and oxidative stress were higher in T2DN patients than those in the control (Fig. 1D and F), while Sirt1 expression was lower (Fig. 1E, $\mathrm{p}<0.05$ for both comparisons). Further univariate correlation analysis showed that renal MIOX expression positively associated with ROS productions (Fig. 2D, $r$ $=0.604$ ), HbA1c (Fig. 2F, $r=0.698$ ), logUACR (Fig. $2 \mathrm{G}, \mathrm{r}=0.633$ ), triglyceride (Fig. $2 \mathrm{H}, \mathrm{r}=$ 0.576 ) and SBP (Fig. 2I, $r=0.556$ ), while inversely related to renal Sirt1 expression (Fig. 2E, $r=-0.562)$.

Table 2. Clinical characteristics of the patients with MCD and T2DN. Abbreviations are as shown in Table 1

\begin{tabular}{|c|c|c|c|}
\hline Parameter & MCD $(n=15)$ & T2DN (n=15) & p-value \\
\hline Age (y) & $47.1 \pm 6.7$ & $48.7 \pm 5.0$ & 0.470 \\
\hline Course of disease (y) & $7.9 \pm 2.3$ & $9.5 \pm 1.1$ & 0.486 \\
\hline $\operatorname{Sex}(m / f)$ & $7 / 8$ & $6 / 9$ & 0.50 \\
\hline BMI $\left(\mathrm{kg} / \mathrm{m}^{2}\right)$ & $22.8 \pm 3.2$ & $23.9 \pm 2.0$ & 0.246 \\
\hline $\mathrm{FBG}(\mathrm{mmol} / \mathrm{l})$ & $4.3 \pm 0.8$ & $8.3 \pm 2.3$ & $<0.01$ \\
\hline $\mathrm{HbA1c} \%(\mathrm{mmol} / \mathrm{mol})$ & $\begin{array}{c}5.4 \pm 0.6 \\
(30.2 \pm 16.6)\end{array}$ & $\begin{array}{c}9.5 \pm 2.0 \\
(79.9 \pm 22.1)\end{array}$ & $<0.01$ \\
\hline SBP (mmHg) & $111.1 \pm 9.8$ & $110.8 \pm 8.5$ & 0.290 \\
\hline $\mathrm{DBP}(\mathrm{mmHg})$ & $72.7 \pm 7.7$ & $69.9 \pm 9.1$ & 0.058 \\
\hline Serum albumin $(\mathrm{g} / \mathrm{L})$ & $32.3 \pm 2.7$ & $30.1 \pm 7.7$ & 0.316 \\
\hline Triglyceride (mmol/L) & $2.0 \pm 1.4$ & $2.0 \pm 1.0$ & 0.938 \\
\hline Total cholesterol $(\mathrm{mmol} / \mathrm{l})$ & $6.7 \pm 1.2$ & $5.3 \pm 1.6$ & 0.011 \\
\hline eGFR (ml/min/1.73 m²) & $111.3 \pm 11.9$ & $88.9 \pm 9.4$ & $<0.01$ \\
\hline Serum creatinine $(\mu \mathrm{mol} / \mathrm{l})$ & $116.7 \pm 17.3$ & $139.5 \pm 40.0$ & 0.060 \\
\hline Uric acid (umol/L) & $290.4 \pm 84.8$ & $295.9 \pm 64.7$ & 0.844 \\
\hline UACR & $880(280-1580)$ & $466.5(354.3-770.8)$ & $<0.01$ \\
\hline Sirt1 (ng/ml) & $10.2 \pm 3.1$ & $4.5 \pm 2.9$ & $<0.01$ \\
\hline uKIM-1/urine $\mathrm{Cr}$ (ng/mg) & $0.5(0.4-0.9)$ & $5.5(2.9-6.7)$ & $<0.01$ \\
\hline uNGAL/urine $\mathrm{Cr}$ (pg/mg) & $6.6(5.0-16.1)$ & $14.5(7.3-64.9)$ & $<0.01$ \\
\hline sMIOX $(\mathrm{pg} / \mathrm{ml})$ & $197.0 \pm 64.0$ & $1570.1 \pm 376.4$ & $<0.01$ \\
\hline uMIOX/urine $\mathrm{Cr}(\mathrm{pg} / \mathrm{mg})$ & $248.0 \pm 45.4$ & $1654.1 \pm 376.6$ & $<0.01$ \\
\hline
\end{tabular}




\section{Kidney Blood Pressure Research}

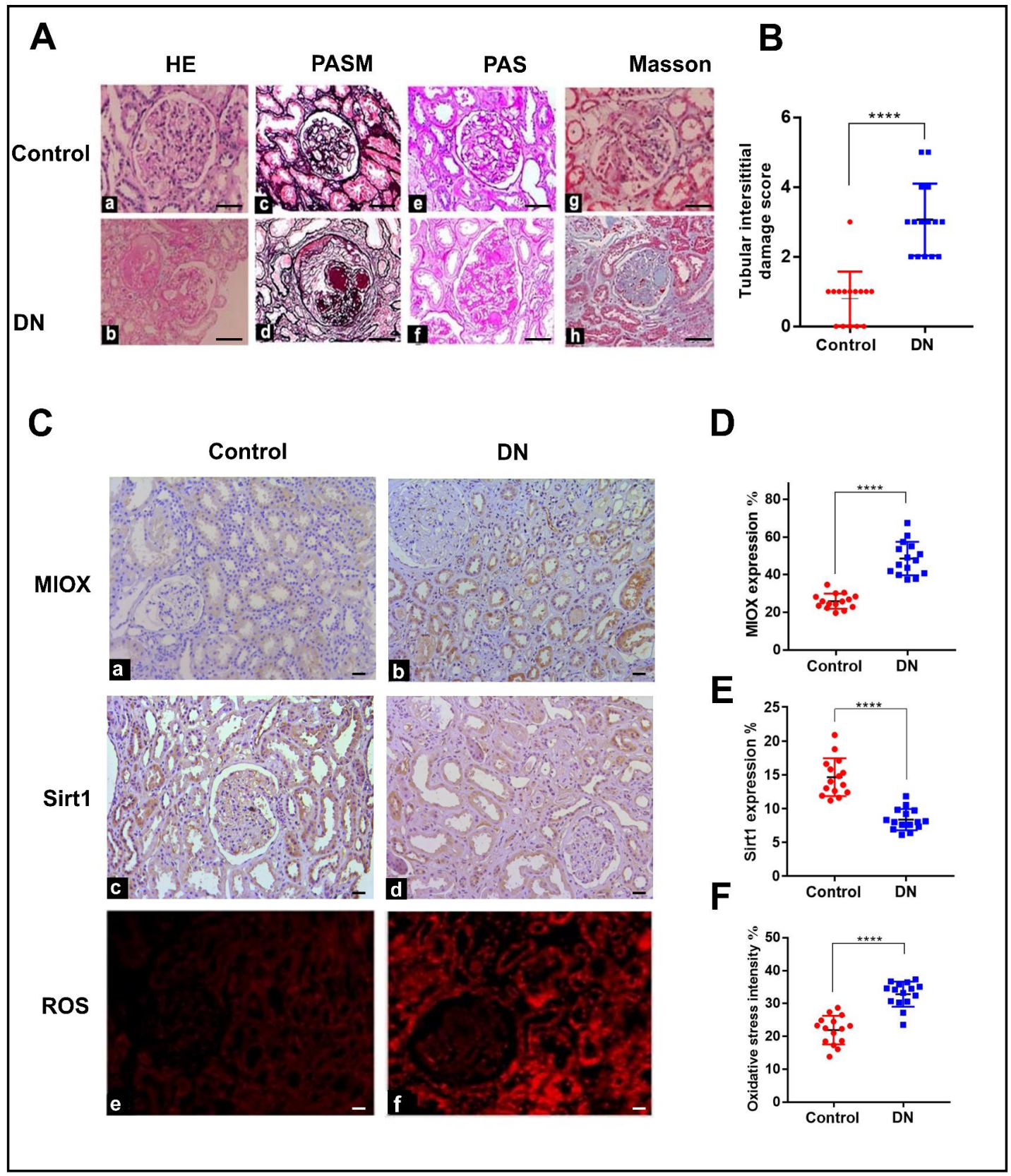

Fig. 1. Assessment of renal pathology, tubular damage score, the expression of MIOX, Sirt1 and ROS generation in patients with DN. (A) Pathology of renal biopsies from DN $(n=15)$ and control groups $(n=15)$ was assessed by HE, PAS, PASM and Masson staining; scale bars: $50 \mu \mathrm{m}$. (B) Tubular interstitial damage score. (C) IHC staining with MIOX antibody (a and b), Sirt1 antibody (c and d) and DHE staining (e and f). A notable increase in MIOX expression and ROS production was seen in the kidneys of DN patients compared to the control ( $\mathrm{b}$ vs a and f vs e). In contrast, the expression of Sirt1 was decreased compared to the control ( $d$ vs c); scale bars: $25 \mu \mathrm{m}$. (D, E and F) Semiquantitative analysis of MIOX, Sirt1 expression and the fluorescence intensity of DHE. Values are means \pm SD. ${ }^{* * * *} \mathrm{p}<0.0001$ vs. control. 


\section{Kidney \\ Blood Pressure Research}

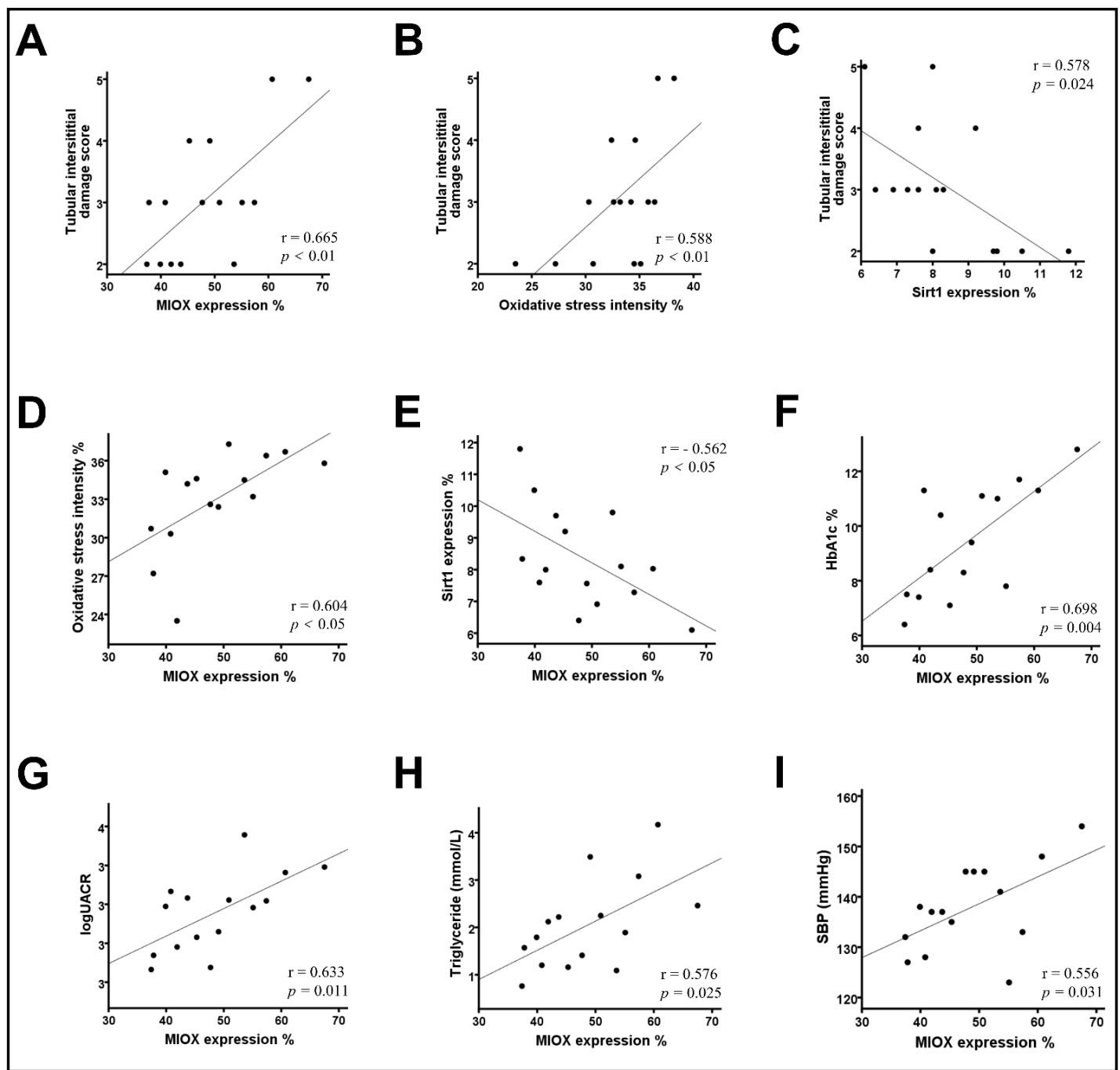

Fig. 2. Correlation analysis among renal MIOX expression, Sirt1 expression, ROS production, tubular interstitial damage and various clinical parameters in patients with DN. Scatter plots representing the relationship among tubular interstitial damage, renal MIOX expression (A) $(r=0.665 ; p<0.01)$, renal ROS level (B) ( $r=0.588 ; p<0.01)$ and renal Sirt1 expression (C) ( $r=-0.578 ; p=0.024)$. D and E represent the relationship between renal MIOX expression and relative renal ROS $(r=0.604 ; p<0.05)$ as well as Sirt1 expression ( $r=-0.562 ; \mathrm{p}<0.05)$. G-I represent the significant relationship between renal MIOX expression and HbA1c $(r=0.698 ; p=0.04)$, $\operatorname{logUACR}(r=0.633 ; p=0.011)$, triglyceride $(r=0.576 ; p=0.025)$ and SBP $(r=$ $0.556 ; \mathrm{p}=0.031)$.

The concentration of MIOX in urine and serum increased while Sirt1 decreased in patients with T2DM

Serum and urinary MIOX levels in patients of the three diabetic groups and healthy control group are shown in Table 1. The MIOX levels in serum and urine in T2DM patients were significantly higher than those in the health controls (Fig. 3, p <0.01). Furthermore, in serum and urine, the concentration of MIOX in the normoalbuminuric, microalbuminuric, and macroalbuminuric groups were gradually increased with the increase in UACR (Fig. 3, $\mathrm{p}<0.01$ ). In addition, in the same group, MIOX levels in the urine were statistically higher than those in the serum except for in the macroalbuminuric group. Moreover, differences in the levels of serum Sirt1 according to UACR are shown in Table 1. Serum levels of Sirt1 in the diabetic groups were significantly decreased compared to the healthy subjects, 


\section{Kidney \\ Blood Pressure \\ Research}

A

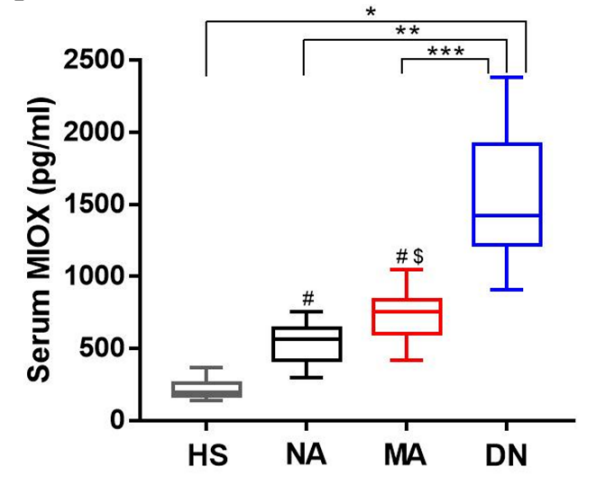

B

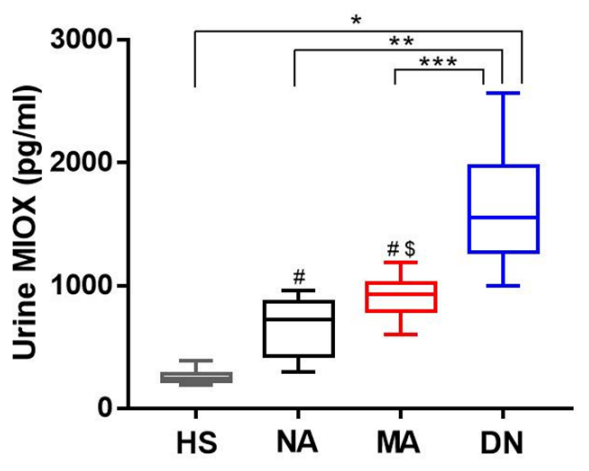

Fig. 3. MIOX levels in the serum and urine of T2DM patients and healthy subjects. (A) ELISA showed that the serum MIOX levels in healthy subjects, the normoalbuminuric group (NA), the microalbuminuric group (MA) and the diabetic nephropathy group (DN group), (B) and the urinary MIOX level in each group. Values are means \pm SD, ${ }^{\#}$ p vs. controls; ${ }^{\$}$ p vs. NA; ${ }^{*}$ p vs. controls; ${ }^{* *}$ p vs. NA; ${ }^{* * *}$ p vs. MA. $p<0.001$.

and decreased with the increase of UACR in normoalbuminuric, microalbuminuric and macroalbuminuric groups $(\mathrm{p}<0.01)$. We also detected the classical tubular damage markers KIM-1and NGAL (Table 1), and they indicated that the urine levels of KIM-1 and NGAL were significantly higher in the macroalbuminuric group than in the control, normoalbuminuria and microalbuminuria groups but were not significantly different between control and normoalbuminuric groups.

sMIOX and UMIOX levels were correlated with various clinical parameters in T2DM patients

To further analyze the concentration of MIOX in the serum or urine of patients with T2DM, a correlation analysis was carried out to assess whether there was an association with the development of T2DN. The results showed that sMIOX was associated positively with fasting blood glucose (Fig. 4A, $r=0.368$ ), HbA1c (Fig. 4B, $r=0.588$ ), serum creatinine (Fig. 4E, $r=0.754$ ), and $\log (\mathrm{UACR}$ ) (Fig. 4F, $r=0.770$ ), while inversely with serum albumin (Fig. 4C, $r=-0.454$ ) and eGFR (Fig. 4D, $r=-0.604$ ). However, there was no obvious correlation with age, BMI, diabetes duration, total cholesterol, SBP or DBP. Similar results were also observed in the relationship between urinary MIOX concentrations and clinical parameters (Fig. 5A-F). Furthermore, the above parameters that were found to be positively correlated with sMIOX at univariate analysis were introduced into a multivariate model using sMIOX as a dependent variable; Significance was only maintained for the correlation between sMIOX and HbA1c $(\beta=0.289)$, Scr $(\beta=0.343)$, and $\operatorname{logUACR}(\beta=0.369 ; \mathrm{p}<0.05$ for all). Similarly, in multivariate analysis, uMIOX was found to be correlated with $\operatorname{HbA1c}(\beta=0.359)$, Scr $(\beta=$ $0.212)$, and $\log$ UACR $(\beta=0.522 ; \mathrm{p}<0.05$ for all). All of the above coefficients are included in Table 3. 


\section{Kidney \\ Blood Pressure \\ Research}

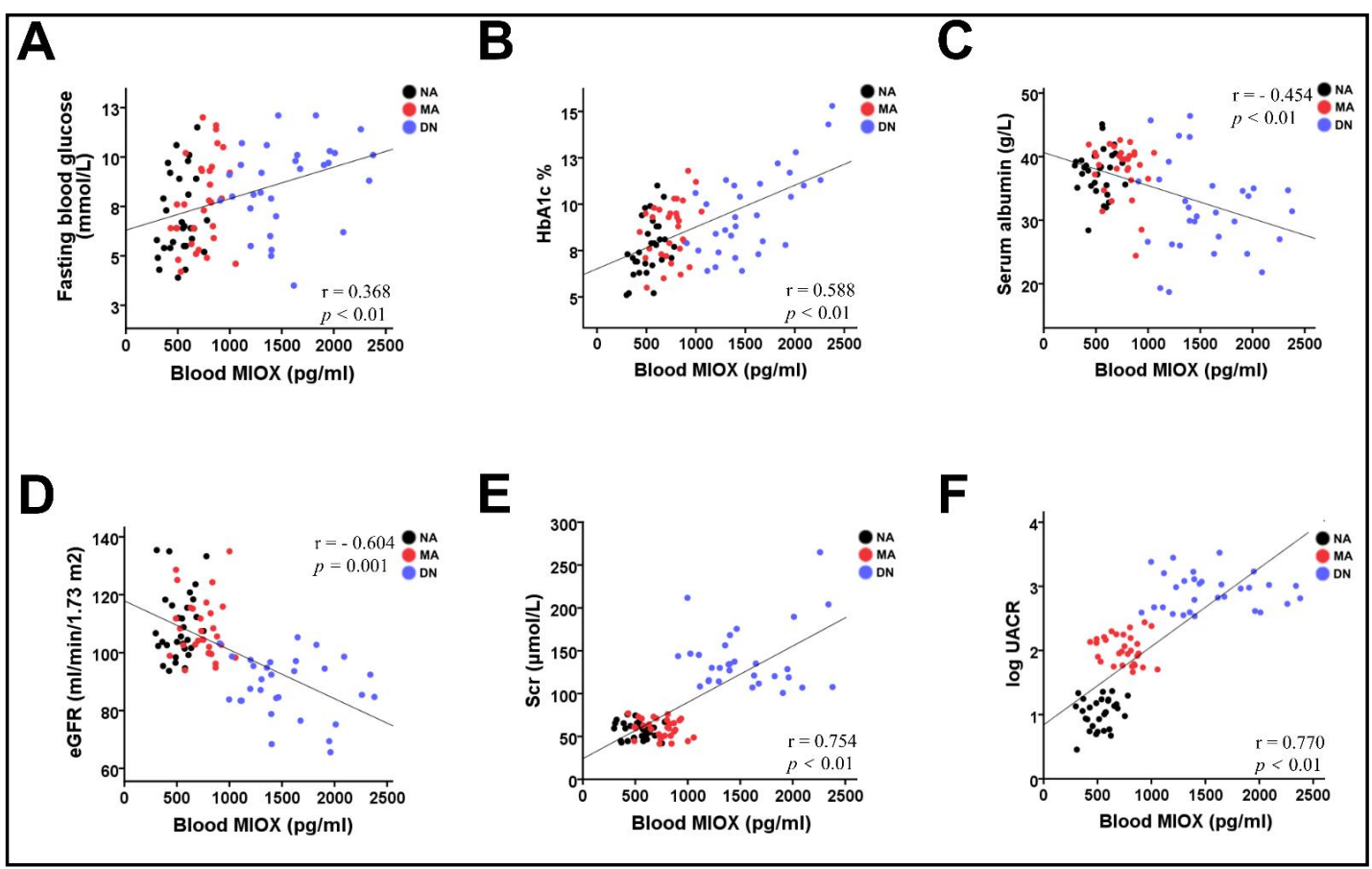

Fig. 4. Univariate analysis of sMIOX and clinical parameters in patients with T2DM. Serum MIOX correlated positively with fasting blood glucose (A) ( $r=0.368$; $p<0.01)$, HbA1c (B) ( $r=0.588 ; p<0.01)$, serum albumin (C) $(r=-0.454 ; p<0.01)$, eGFR (D) $(r=-0.604 ; p=0.001)$, Scr (E) ( $r=0.754 ; p<0.01)$, and logUACR (F) (r=0.770; $\mathrm{p}<0.01$ ) respectively.

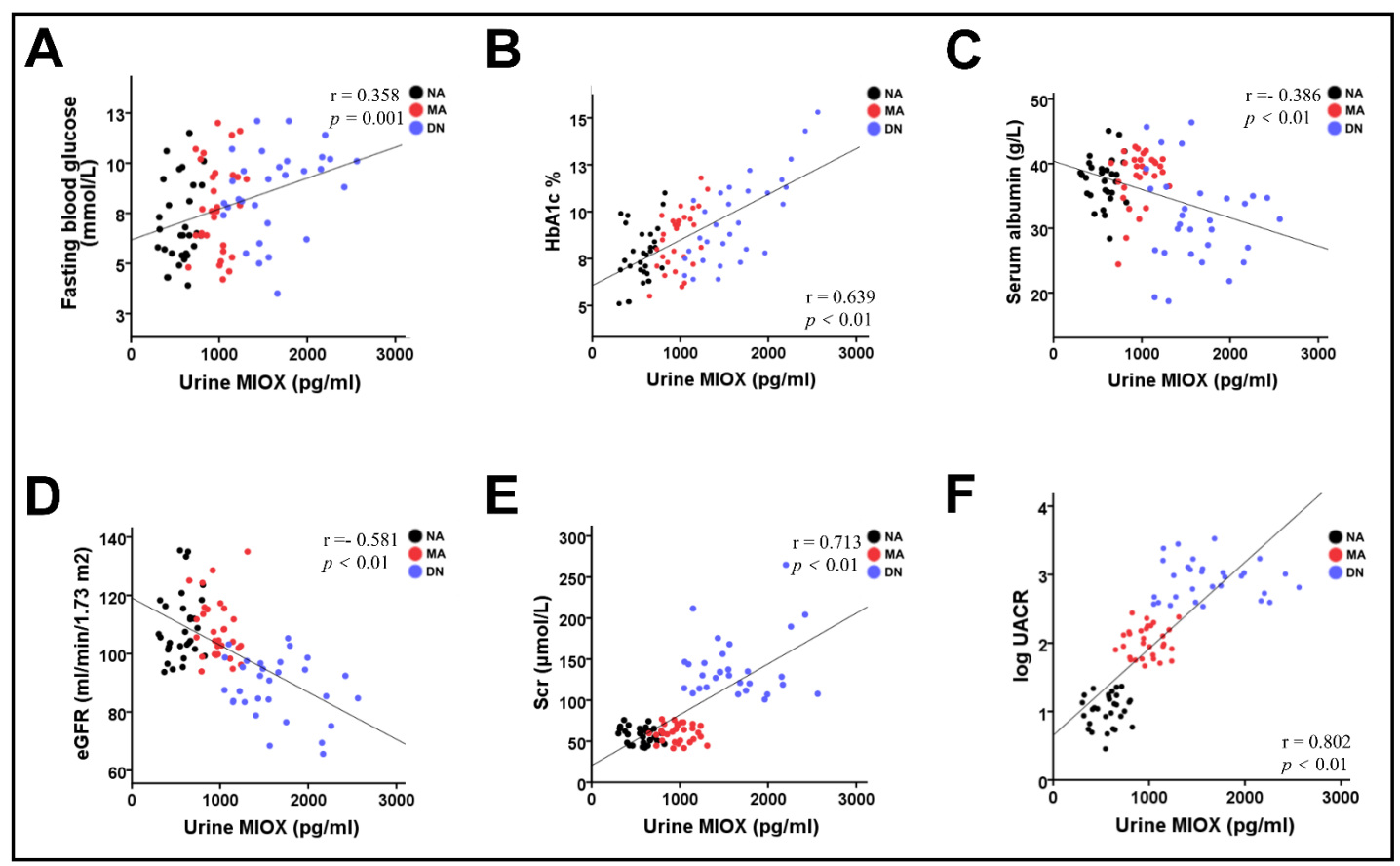

Fig. 5. Univariate analysis of uMIOX and clinical parameters in patients with T2DM. Urinary MIOX correlated positively with fasting blood glucose $(A)(r=0.358 ; p=0.001)$, HbA1c (B) ( $r=0.639 ; p<0.01)$, serum albumin (C) $(r=-0.386 ; p<0.01)$, eGFR (D) $(r=-0.581 ; p<0.01)$, serum creatinine (E) $(r=0.713 ; p<0.01)$, and $\operatorname{logUACR}$ (F) $(\mathrm{r}=0.802 ; \mathrm{p}<0.01)$ respectively. 
Kidney Blood Press Res 2018;43:1772-1785

\begin{tabular}{l|cl}
\hline DOI: 10.1159/000495635 & $\odot 2018$ The Author(s). Published by S. Karger AG, Basel \\
\hline
\end{tabular} Published online: 1 December 2018 www.karger.com/kbr

\section{Kidney Blood Pressure Research}

ROC analysis of SMIOX and $\mathrm{UMIOX}$

ROC analysis was performed to define the diagnostic profile of sMIOX and UMIOX in screening patients with T2DM among all participants with normal $+\quad$ normoalbuminuric group). To this end, sMIOX exhibited a very good diagnostic profile, depicting an AUC of 0.981 (CI 0.9070.999) with an optimal cutoff value of $299.9 \mathrm{pg} /$ ml (sensitivity 96.67 \%; specificity $86.67 \%$ ) (Table 4 and Fig. 6). Additionally, uMIOX exhibited a good diagnostic profile, showing an AUC of 0.986 (CI 0.9141.000 ) and an optimal cutoff value of $323.1 \mathrm{ng} /$ ml (sensitivity 93.33\%; specificity $96.67 \%$ ) (Table 5 and Fig. 6). However, there is no difference between these two areas $(p=0.7422)$. UACR (i.e., healthy controls

Table 3. Univariate correlation analysis of renal MIOX, sMIOX and uMIOX with clinical parameters. Abbreviations are as shown in Table 1

\begin{tabular}{lcccccc}
\hline \multirow{2}{*}{ Parameter } & \multicolumn{2}{c}{ Renal MIOX } & \multicolumn{2}{c}{ Serum MIOX } & \multicolumn{2}{c}{ Urinary MIOX } \\
& $\mathrm{r}$ & $\mathrm{p}$-value & $\mathrm{r}$ & $\mathrm{p}$-value & $\mathrm{r}$ & $\mathrm{p}$-value \\
\hline Age $(\mathrm{y})$ & 0.017 & 0.951 & 0.054 & 0.613 & 0.017 & 0.874 \\
Diabetes duration (y) & 0.004 & 0.989 & 0.064 & 0.548 & 0.064 & 0.548 \\
BMI $\left(\mathrm{kg} / \mathrm{m}^{2}\right.$ ) & -0.004 & 0.989 & 0.130 & 0.223 & 0.299 & 0.111 \\
FBG $(\mathrm{mmol} / \mathrm{l})$ & 0.303 & 0.272 & 0.368 & $<0.01$ & 0.358 & 0.001 \\
HbA1c \% & 0.698 & 0.004 & 0.588 & $<0.01$ & 0.639 & $<0.01$ \\
SBP $(\mathrm{mmHg})$ & 0.556 & 0.031 & 0.184 & 0.083 & 0.202 & 0.056 \\
DBP $(\mathrm{mmHg})$ & 0.015 & 0.958 & 0.006 & 0.955 & -0.011 & 0.915 \\
Alb $(\mathrm{g} / \mathrm{l})$ & 0.052 & 0.855 & -0.454 & $<0.01$ & -.386 & $<0.01$ \\
Total cholesterol $(\mathrm{mmol} / \mathrm{l})$ & 0.017 & 0.953 & 0.205 & 0.053 & 0.188 & 0.076 \\
eGFR (ml/min/1.73 m $\left.{ }^{2}\right)$ & -0.469 & 0.078 & -0.604 & $<0.01$ & -0.581 & $<0.01$ \\
Serum creatinine $(\mu \mathrm{mol} / \mathrm{l})$ & 0.488 & 0.065 & 0.754 & $<0.01$ & 0.713 & $<0.01$ \\
logUACR & 0.633 & 0.011 & 0.770 & $<0.01$ & 0.802 & $<0.01$ \\
\hline
\end{tabular}

Table 4. ROC analysis of sMIOX values. Best cutoff value for the identification of diabetic patients among subjects with normal albumin excretion (health subjects and normoalbuminuric diabetic patients): $[299.9 \mathrm{pg} / \mathrm{ml}$. Area under the ROC curve $=0.981 ; \mathrm{SE}=0.0121 ; 95 \%$ $\mathrm{CI}=0.907-0.999 .+$ LR Positive likelihood ratio; -LR negative likelihood ratio. ${ }^{\text {a }}$ Best sMIOX cutoff value

\begin{tabular}{lcccc}
\hline $\begin{array}{l}\text { sMIOX } \\
\text { value pg/ml }\end{array}$ & $\begin{array}{c}\text { Sensitivity } \\
(95 \% \mathrm{CI})\end{array}$ & $\begin{array}{c}\text { Specificity } \\
(95 \% \mathrm{CI})\end{array}$ & $+\mathrm{LR}$ & -LR \\
\hline$\geq 141.2$ & $100.00(88.4-100.0)$ & $0.00(0.0-11.6)$ & 1.00 & \\
$>281.3$ & $100.00(88.4-100.0)$ & $80.00(61.4-92.3)$ & 5.00 & 0.00 \\
$>298.3$ & $96.67(82.8-99.9)$ & $80.00(61.4-92.3)$ & 4.83 & 0.042 \\
$>299.9 \mathrm{a}$ & $96.67(82.8-99.9)$ & $86.67(69.3-96.2)$ & 7.25 & 0.038 \\
$>307.8$ & $93.33(77.9-99.2)$ & $86.67(69.3-96.2)$ & 7.00 & 0.077 \\
$>308.4$ & $93.33(77.9-99.2)$ & $90.00(73.5-97.9)$ & 9.33 & 0.074 \\
$>327.2$ & $86.67(69.3-96.2)$ & $90.00(73.5-97.9)$ & 8.67 & 0.15 \\
$>360.3$ & $86.67(69.3-96.2)$ & $96.67(82.8-99.9)$ & 26.00 & 0.14 \\
$>361.3$ & $83.33(65.3-94.4)$ & $96.67(82.8-99.9)$ & 25.00 & 0.17 \\
$>365.4$ & $83.33(65.3-94.4)$ & $100.00(88.4-100.0)$ & & 0.17 \\
$>754.8$ & $0.00(0.0-11.6)$ & $100.00(88.4-100.0)$ & & 1.00 \\
\hline
\end{tabular}

Fig. 6. ROC analysis of sMIOX and uMIOX in health subjects and normoalbuminuric patients. The AUCs for sMIOX and uMIOX were 0.981 (CI $0.907-0.999$ ) and 0.986 (CI $0.914-1.000)$, respectively. The difference between the two areas was not significant ( $p$ $=0.7422$ ). For sMIOX the best cutoff value for the identification of diabetes was found to be $299.9 \mathrm{pg} / \mathrm{mL}$ with a sensitivity of 96.6 (CI 82.8 .7 - 99.9) and a specificity of 86.6 (CI 69.3 - 96.2), whereas for UMIOX it was $323.1 \mathrm{pg} / \mathrm{mL}$ with a sensitivity of 93.3 (CI $77.9-99.2$ ) and a specificity of 96.6 (CI $82.8-99.9$ ).

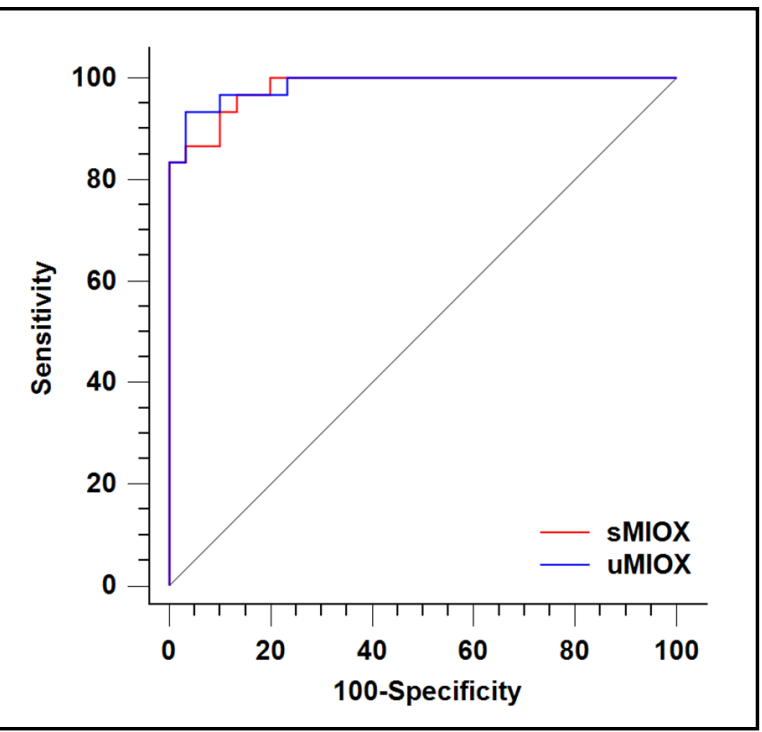




\section{Kidney Blood Pressure Research}

\section{Discussion}

The investigation described in the previous section highlights three major findings. First, MIOX expression was found to be increased in renal biopsy tissues of T2DN patients and was positively correlated with tubular injury and renal oxidative stress, while negatively associated with Sirt1 expression. Second, serum and urinary MIOX levels were also significantly increased in patients with T2DM, even before the diagnosis of microalbuminuria, representing an early biomarker of "normoalbuminuric" DN. Third, sMIOX and uMIOX showed an optimal diagnostic power in identifying the presence of tubular damage among all normoalbuminuric subjects with T2DM.

By convention, the general progression of DN starts with increasing UACR, which is the most widely recognized early diagnosis marker [2]. However, in some patients with T2DM this is usually accompanied by coronary atherosclerosis, and the UACR is correlated significantly with the severity of coronary atherosclerosis [21]. Thus, we believe that UACR is prone to a non-optimal biomarker for revealing early tubular damage in DN. In addition, many studies have demonstrated that tubular damage plays a key role in the progression of DN and the extent of damage to this compartment correlated much better with the decline of renal function (e.g., decrease in the eGFR) than does damage to the glomerular tissue [22]. For example, urinary proteomic analysis of T1DM patients with normoalbuminuria and microalbuminuria has highlighted several tubule-related proteins, whose expression were strictly correlated to $\mathrm{HbA1c}$ and microalbuminuria levels [23]. Furthermore, in clinical practice, we usually find some diabetic patients who manifest with normal UACR but have renal insufficiency and pathological changes that majorly present as tubulointerstitial damage [2]. Now it has been referred to as normoalbuminuric diabetic kidney disease (NADKD) [24]. However, there is still a lack of potential biomarkers to clinically screen for NADKD.

In vivo and in vitro studies have demonstrated that the MIOX expression was confined to the proximal tubular cells, and its expression was up-regulated in HK-2 cells (a human proximal tubular cell line) and LLC-PK1 (porcine) when exposed to high-glucose $[15,17]$. In the present study, we also found that MIOX expression was significantly elevated in renal biopsy tissues from T2DN patients and predominantly expressed in the renal tubular cells (Fig. 1A b vs a). Moreover, the increased MIOX expression was correlated positively with tubular interstitial damage (Fig. 2A), indicating that abnormal increased expression of MIOX in the kidney may participate in the pathogenesis of DN. Interestingly, we also found that increased MIOX expression correlated positively with HbA1c (Fig. 2F), which was in line with what Nayak and Lu have reported: that MIOX expression was in proportion to serum glucose concentration in $\mathrm{db} / \mathrm{db}$ mice and rats with $\mathrm{DN}[14,25]$.

Overproduction of ROS is a common event in hyperglycemia situation [26]. Many studies by Dr. Kanwar and other groups have shown that increased MIOX expression could induce ROS generation in diabetic kidneys $[15,27]$. In the current study, we demonstrated that ROS production was significantly increased in renal biopsy tissues of patients with T2DN as identification of diabetic patients among subjects with normal albumin excretion (health subjects and normoalbuminuric diabetic patients): $[323.1 \mathrm{pg} / \mathrm{ml}$. Area under the ROC curve $=0.986 ; \mathrm{SE}=0.0105 ; 95 \% \mathrm{CI}$

\begin{tabular}{lcccc}
\hline $\begin{array}{l}\text { uMIOX } \\
\text { value } \mathrm{pg} / \mathrm{ml}\end{array}$ & $\begin{array}{c}\text { Sensitivity } \\
(95 \% \mathrm{CI})\end{array}$ & $\begin{array}{c}\text { Specificity } \\
(95 \% \mathrm{CI})\end{array}$ & $+\mathrm{LR}$ & -LR \\
\hline$\geq 187.7$ & $100.00(88.4-100.0)$ & $0.00(0.0-11.6)$ & 1.00 & \\
$>279.2$ & $100.00(88.4-100.0)$ & $76.67(57.7-90.1)$ & 4.29 & 0.00 \\
$>294.1$ & $96.67(82.8-99.9)$ & $76.67(57.7-90.1)$ & 4.14 & 0.043 \\
$>309.8$ & $96.67(82.8-99.9)$ & $90.00(73.5-97.9)$ & 9.67 & 0.037 \\
$>319.8$ & $93.33(77.9-99.2)$ & $90.00(73.5-97.9)$ & 9.33 & 0.074 \\
$>323.1^{\mathrm{a}}$ & $93.33(77.9-99.2)$ & $96.67(82.8-99.9)$ & 28.00 & 0.069 \\
$>379.9$ & $83.33(65.3-94.4)$ & $96.67(82.8-99.9)$ & 25.00 & 0.17 \\
$>389.6$ & $83.33(65.3-94.4)$ & $100.00(88.4-100.0)$ & & 0.17 \\
$>961.8$ & $0.00(0.0-11.6)$ & $100.00(88.4-100.0)$ & & 1.00 \\
\hline
\end{tabular}




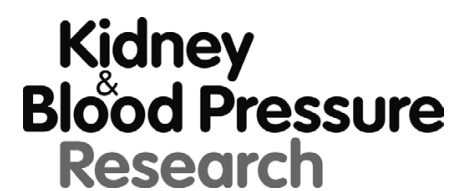
\begin{tabular}{l}
\hline Kidney Blood Press Res 2018;43:1772-1785 \\
\begin{tabular}{l|l} 
DOI: 10.1159/000495635 & C 2018 The Author(s). Published by S. Karger AG, Basel \\
Published online: 1 December 2018 & www.karger.com/kbr
\end{tabular}
\end{tabular}

detected by DHE staining (Fig. 1B f vs e). Furthermore, the increased ROS production was correlated positively with tubular interstitial damage (Fig. 2B) and renal MIOX expression (Fig. 2D), confirming that the increased MIOX expression related to enhanced ROS production in the kidneys of patients with T2DN.

In addition, Sirt1, an $\mathrm{NAD}^{+}$-dependent deacetylase, functions as sensor to detect the concentration of $\mathrm{NAD}^{+}$and regulates metabolic changes by making histones, nuclear transcriptional factors and enzymes deacetylation [28]. Recently, we confirmed that Glucuronate-Xylulose (G-X) pathway was involved in the metabolism of MI in the proximal tubules of the kidney and MIOX served as the crucial enzyme [16]. Two different steps of the G-X pathway consume a large amount of $\mathrm{NAD}^{+}$. As a result, intracellular $\mathrm{NAD}^{+}$depletion induced Sirt1 inactivation and lead to renal damage [28]. Likewise, consistent with what had been found in type 2 diabetic rats [29], our study found that renal Sirt1 expression was downregulated in patients with T2DN (Fig. 1B d vs c). We also found that Sirt1 expression was inversely correlated with tubular interstitial damage (Fig. 2C) and renal MIOX expression (Fig. $2 \mathrm{E}$ ), indicating that upregulated renal MIOX expression induces the inactivation of Sirt1and is involved in the development of tubular injury. Taken together, renal tubulointerstitial injury is probably modulated by MIOX during the progression of T2DN.

In this regard, renal MIOX expression may serve as a marker for tubular damage in renal biopsy tissues of T2DN patients, but it may not be practical because collecting kidney specimens during the early stages of DN could be a major impediment. Therefore, it is important for nephrologists to identify a sensitive biomarker to screen for tubular injury, especially for patients with normoalbuminuria.

Thus, we examined the MIOX levels in the serum and urine of T2DM patients with different UACR. We then performed an ELISA to measure the serum/urine MIOX concentration and Spearman's correlation to access the relationships between MIOX level and various clinical parameters. Compared to the controls, the levels of sMIOX and UMIOX were significantly increased in normoalbuminuric diabetic patients whose serum creatinine and UACR were in the normal range. The urine levels of KIM-1 and NGAL were not significantly different between the control and normoalbuminuric groups (Table 1), suggesting that KIM-1 and NGAL may not function as early maker of diabetic nephropathy. The reason for these results may be that our study subjects were comprised of T2DN with eGFR of more than $60 \mathrm{~mL} /$ $\min / 1.73 \mathrm{~m}^{2}$. We also found that the levels of sMIOX or UMIOX in patients with T2DM were gradually elevated with the increase in UACR (Fig. 3). Although there is a positive correlation between serum/urinary MIOX and UACR in diabetic patients, they are not increased in MCD patients with massive albuminuria, suggesting the level of MIOX is independent of albuminuria. These data suggest that the elevation of MIOX in serum or urine preceded the elevation of UACR, indicating its use as a potential diagnostic biomarker for early tubular injury in T2DN.

It is known that poor glycemic control, hypertension, and hyperlipidemia are significant risk factors for the progression of DN [30]. Thus, we performed univariate correlation analysis and found that renal MIOX expression associated positively with logUACR (Fig. 2G), triglyceride levels (Fig. 2H) and SBP (Fig. 2I). Likewise, sMIOX and uMIOX were directly correlated with fasting blood glucose (Fig. 4A and Fig. 5A), HbA1c (Fig. 4B and Fig. 5B), Scr (Fig. 4E and Fig. 5E), and logUACR (Fig. 4F and Fig. 5F), while inversely related to serum albumin (Fig. 4C and Fig. 5C) and eGFR (Fig. 4D and Fig. 5D). Furthermore, ROC analysis showed that sMIOX or uMIOX exhibited an optimal diagnostic power in identifying the presence of tubular damage among all normoalbuminuric participants (Fig. 6). Another study has demonstrated that the expression of MIOX is not confined to the kidney and can also be detected in the retina and human lens epithelial cells which are also susceptible to the complications of DM[13]. However, the kidney is the only major organ where the MIOX protein is expressed at detectable levels. Thus, we conclude that the renal tissues are the major resource of MIOX in patients with DM. Consequently, these data indicate that the MIOX 


\section{Kidney Blood Pressure Research}

levels in the serum or urine may be a predictive marker of the progression of renal tubular interstitial damage in patients with T2DM.

There are limitations in this study, since it was a single-center and cross-sectional study and the pathogenesis of diabetic nephropathy is complex, and is not only simply involved in tubular injury but also the damage and reduction of podocytes. Therefore, MIOX combined with other diagnostic markers or indices might have more predictive value for the early diagnosis of DN, and further study is needed.

\section{Conclusion}

The current study demonstrated for the first time that MIOX expression is up-regulated in renal biopsy tissues of patients with T2DN. Furthermore, it was shown that MIOX levels are increased in the serum and urine of patients with T2DM. Thus, sMIOX or uMIOX may be new biomarkers for identifying early tubular damage in T2DN and a potential diagnostic parameter for NADKD.

\section{Acknowledgements}

This work was supported by grants from the National Natural Sciences Foundation of China (81730018), National Basic Research Program of China (2016YFC1305501 and 2018YFC1314002). We would like to acknowledge the help and support of the Department of Nephology, Second Xiangya Hospital, Central South University, Changsha, the China and Departments of Pathology \&Medicine, Northwestern University, Chicago, USA. We also thank Dr. Kanwar, for his helpful advice on the manuscript.

\section{Disclosure Statement}

The authors declare that they have no competing interests and nothing to disclose.

\section{References}

1 Kanwar YS, Sun L, Xie P, Liu FY, Chen S: A glimpse of various pathogenetic mechanisms of diabetic nephropathy. Annu Rev Pathol 2011;6:395-423.

2 Chen C, Wang C, Hu C, Han Y, Zhao L, Zhu X, Xiao L, Sun L: Normoalbuminuric diabetic kidney disease. Front Med 2017;11:310-318.

-3 MacIsaac RJ, Tsalamandris C, Panagiotopoulos S, Smith TJ, McNeil KJ, Jerums G: Nonalbuminuric renal insufficiency in type 2 diabetes. Diabetes Care 2004;27:195-200.

-4 Cooper ME, Gilbert RE, Epstein M: Pathophysiology of diabetic nephropathy. Metabolism 1998;47:3-6.

5 Thomas MC, Burns WC, Cooper ME: Tubular changes in early diabetic nephropathy. Adv Chronic Kidney Dis 2005;12:177-186.

6 Wolf G: Cell cycle regulation in diabetic nephropathy. Kidney Int Suppl 2000;77:S59-66.

7 Pfleiderer S, Zimmerhackl LB, Kinne R, Manz F, Schuler G, Brandis M: Renal proximal and distal tubular function is attenuated in diabetes mellitus type 1 as determined by the renal excretion of alpha 1-microglobulin and Tamm-Horsfall protein. Clin Investig 1993;71:972-977.

8 Xiao L, Wang M, Yang S, Liu F, Sun L: A glimpse of the pathogenetic mechanisms of Wnt/beta-catenin signaling in diabetic nephropathy. Biomed Res Int 2013;2013:987064.

-9 Gilbert RE, Cooper ME: The tubulointerstitium in progressive diabetic kidney disease: more than an aftermath of glomerular injury? Kidney Int 1999;56:1627-1637. 


\section{Kidney \\ Bloód Pressure Research}

\begin{tabular}{l|l}
\hline Kidney Blood Press Res 2018;43:1772-1785 \\
\hline $\begin{array}{l}\text { DOI: 10.1159/000495635 } \\
\text { Published online: 1 December } 2018\end{array}$ & $\begin{array}{l}\text { ( ) 2018 The Author(s). Published by S. Karger AG, Basel } \\
\text { www.karger.com/kbr }\end{array}$ \\
\hline
\end{tabular}

Sun et al.: MIOX a Potential Biomarker For Diabetic Nephropathy

10 Matheson A, Willcox MD, Flanagan J, Walsh BJ: Urinary biomarkers involved in type 2 diabetes: a review. Diabetes Metab Res Rev 2010;26:150-171.

11 Yang Q Dixit B, Wada J, Tian Y, Wallner EI, Srivastva SK, Kanwar YS: Identification of a renal-specific oxidoreductase in newborn diabetic mice. Proc Natl Acad Sci U S A 2000;97:9896-9901.

12 Arner RJ, Prabhu KS, Reddy CC: Molecular cloning, expression, and characterization of myo-inositol oxygenase from mouse, rat, and human kidney. Biochem Biophys Res Commun 2004;324:1386-1392.

13 Arner RJ, Prabhu KS, Krishnan V, Johnson MC, Reddy CC: Expression of myo-inositol oxygenase in tissues susceptible to diabetic complications. Biochem Biophys Res Commun 2006;339:816-820.

14 Nayak B, Xie P, Akagi S, Yang Q, Sun L, Wada J, Thakur A, Danesh FR, Chugh SS, Kanwar YS: Modulation of renal-specific oxidoreductase/myo-inositol oxygenase by high-glucose ambience. Proc Natl Acad Sci U S A 2005;102:17952-17957.

15 Xie P, Sun L, Oates PJ, Srivastava SK, Kanwar YS: Pathobiology of renal-specific oxidoreductase/myo-inositol oxygenase in diabetic nephropathy: its implications in tubulointerstitial fibrosis. Am J Physiol Renal Physiol 2010;298:F1393-1404.

16 Sun L, Dutta RK, Xie P, Kanwar YS: myo-Inositol Oxygenase Overexpression Accentuates Generation of Reactive Oxygen Species and Exacerbates Cellular Injury following High Glucose Ambience: A NEW MECHANISM RELEVANT TO THE PATHOGENESIS OF DIABETIC NEPHROPATHY. J Biol Chem 2016;291:5688-5707.

17 Zhan M, Usman IM, Sun L, Kanwar YS: Disruption of renal tubular mitochondrial quality control by myoinositol oxygenase in diabetic kidney disease. J Am Soc Nephrol 2015;26:1304-1321.

18 Gaut JP, Crimmins DL, Ohlendorf MF, Lockwood CM, Griest TA, Brada NA, Hoshi M, Sato B, Hotchkiss RS, Jain $\mathrm{S}$, Ladenson JH: Development of an immunoassay for the kidney-specific protein myo-inositol oxygenase, a potential biomarker of acute kidney injury. Clin Chem 2014;60:747-757.

19 Zhang Z, Sun L, Wang Y, Ning G, Minto AW, Kong J, Quigg RJ, Li YC: Renoprotective role of the vitamin D receptor in diabetic nephropathy. Kidney Int 2008;73:163-171.

20 Haruna Y, Kashihara N, Satoh M, Tomita N, Namikoshi T, Sasaki T, Fujimori T, Xie P, Kanwar YS: Amelioration of progressive renal injury by genetic manipulation of Klotho gene. Proc Natl Acad Sci U S A 2007;104:2331-2336.

-21 Guo L, Cheng Y, Wang X, Pan Q, Li H, Zhang L, Wang Y: Association between microalbuminuria and cardiovascular disease in type 2 diabetes mellitus of the Beijing Han nationality. Acta Diabetol 2012;49:S65-71.

22 Nath KA: Tubulointerstitial changes as a major determinant in the progression of renal damage. Am J Kidney Dis 1992;20:1-17.

23 Soggiu A, Piras C, Bonizzi L, Hussein HA, Pisanu S, Roncada P: A discovery-phase urine proteomics investigation in type 1 diabetes. Acta Diabetol 2012;49:453-464.

24 Macisaac RJ, Jerums G: Diabetic kidney disease with and without albuminuria. Curr Opin Nephrol Hypertens 2011;20:246-257.

25 Lu Y, Liu C, Miao X, Xu K, Wu X, Liu C: Increased expression of myo-inositol oxygenase is involved in the tubulointerstitial injury of diabetic nephropathy. Exp Clin Endocrinol Diabetes 2009;117:257-265.

-26 Kanwar YS, Wada J, Sun L, Xie P, Wallner EI, Chen S, Chugh S, Danesh FR: Diabetic nephropathy: mechanisms of renal disease progression. Exp Biol Med (Maywood) 2008;233:4-11.

-27 Nayak B, Kondeti VK, Xie P, Lin S, Viswakarma N, Raparia K, Kanwar YS: Transcriptional and posttranslational modulation of myo-inositol oxygenase by high glucose and related pathobiological stresses. J Biol Chem 2011;286:27594-27611.

28 Kume S, Kitada M, Kanasaki K, Maegawa H, Koya D: Anti-aging molecule, Sirt1: a novel therapeutic target for diabetic nephropathy. Arch Pharm Res 2013;36:230-236.

29 Kitada M, Takeda A, Nagai T, Ito H, Kanasaki K, Koya D: Dietary restriction ameliorates diabetic nephropathy through anti-inflammatory effects and regulation of the autophagy via restoration of Sirt1 in diabetic Wistar fatty (fa/fa) rats: a model of type 2 diabetes. Exp Diabetes Res 2011;2011:908185.

- 30 Al-Rubeaan K, Youssef AM, Subhani SN, Ahmad NA, Al-Sharqawi AH, Al-Mutlaq HM, David SK, AlNaqeb D: Diabetic nephropathy and its risk factors in a society with a type 2 diabetes epidemic: a Saudi National Diabetes Registry-based study. PLoS One 2014;9:e88956. 